\title{
Estrategias de Captación de Clientes para una Mype del Sector Comercial ante el Covid-19
}

\section{Customer Attraction Strategies for a SME Company in the Commercial Sector before the Covid-19}

\author{
Odar R. Florián Castillo, MBA. ${ }^{1}$, Liz A. Dominguez Alcalde, Ingeniera ${ }^{2}$ \\ ${ }^{1}$ Universidad Privada del Norte, Perú, odar.florian@upn.edu.pe \\ ${ }^{2}$ Ingenieria Empresarial, Universidad Privada del Norte, Perú, ldominguezalcalde@gmail.com
}

\begin{abstract}
The objective of the research was to formulate strategies aimed at attracting clients for a Mype of the commercial sector dedicated to the commercialization of basic food products. The study was prospective. A reliable questionnaire (Cronbach's Alpha of 0.868) and validated by expert judgment was applied to 20 frequent clients sampled for convenience. The main problems encountered were: the lack of use of technologies, the lack of knowledge about strategies and the strong competition that the company faces. Finally, the internal and external analysis strategies were formulated: to provide additional services to clients by frequency of purchase and recommendations; implementation of digital media for advertising and sales, carry out a campaign of low-demand products, recovering the investment or obtaining slight profits; implement resources to avoid contagion in the sale process (seller - customer), implement price or service inquiries through calls or the use of WhatsApp to avoid direct physical contact with customers and possible infections.

Keywords- Customer's attraction, Commercial sector, Loyalty, Strategies, Covid-19.
\end{abstract}

Resumen- La investigación tuvo por objetivo formular estrategias orientadas a la captación de clientes para una Mype del sector comercial dedicada a la comercialización de productos alimenticios de primera necesidad. El estudio fue de carácter prospectivo. Se aplicó un cuestionario confiable (Alpha de Cronbach de 0.868) y validado por el juicio de expertos a 20 clientes frecuentes muestreados por conveniencia. Los principales problemas encontrados fueron: la falta de uso de tecnologías, la falta de conocimiento sobre estrategias y la fuerte competencia que enfrenta la empresa. Finalmente, del análisis interno y externo se formularon estrategias: Brindar servicios adicionales a clientes por frecuencia de compra y recomendaciones; implementación de medios digitales para publicidady ventas, realizar una campaña de productos de baja demanda, recuperando la inversión u obteniendo ligeras ganancias; implementar recursos para evitar el contagio en el proceso de la venta (vendedor - cliente), implementar las consultas de precios o servicios por medio de llamadas o el uso del WhatsApp para evitar el contacto físico directo con los clientes y posibles contagios.

Palabras clave-Captación de clientes, Sector comercial, Fidelización, Estrategias, Covid-19.

Digital Object Identifier (DOI):

http://dx.doi.org/10.18687/LEIRD2021.1.1.8

ISSN: 2414-6390 ISBN: 978-958-52071-9-6

\section{INTRODUCCIÓN}

El COVID-19 que inició en el 2020, trajo diversas consecuencias, tanto en el aspecto social y económico, como la pérdida de vidas humanas y la quiebra de varias empresas, siendo las más afectadas las MYPES. El aislamiento y falta de planes de contingencia, afecto mucho a diferentes sectores a nivel mundial, generando caos y desesperación en sus ciudadanos [1]. Actualmente, a nivel mundial las diferentes empresas del sector comercial han sido obligadas a buscar varias alternativas para lograr sobrevivir ante la crisis sanitaria y permitir su permanencia en el mercado.

Ciertas empresas optaron por una transformación digital que permitan la captación de clientes, utilizándolo también como medio de ventas, sin embargo, esto conlleva a un equilibrio respecto a las oportunidades de promoción, tema salarial interno y externo y un equilibrio entre familia-labor, además se debe tener en cuenta el tema de costos, ya que este tipo de trasformación conlleva a tener altos costos de inversión a cambio de pocos beneficios [2]. Sin embargo, otras empresas optaron por estrategias de digitalización, como el uso del home office para el apoyo de actividades administrativas y con el uso de algunas plataformas para la comercialización de sus productos [3]. Por otro lado, algunas de ellas, plantearon estrategias como la promoción por medio de canales virtuales y/o tradicionales, aplicación de protocolos de saneamiento, inversión en productos anti-COVID, cambio de horarios e innovación de productos [4], logrando cumplir con las necesidades del público y captando nuevos consumidores.

Si bien es cierto, se sabe que el objetivo de toda empresa es lograr conseguir clientes satisfechos, para así poder lograr su fidelidad, y por otro sus recomendaciones a otros consumidores. De este modo, se puede concluir que la lealtad del consumidor es el objetivo principal del marketing de relaciones, ya que el consumidor no solo volverá a comprarnos productos o servicios, sino que también nos recomendará a su entorno [5].

En América Latina y el Caribe, el comercio internacional obtuvo una pronunciada caída de $23 \%$ en 2020 , más de la crisis financiera de 2009 -cuando disminuyó 21\%- como consecuencia de la pandemia del coronavirus (COVID-19), 
trayendo consigo efectos perjudiciales a la economía de diversos países involucrados [6].

En el contexto peruano, el sector comercio representa alrededor del 11\% del PBI, es decir, 24 mil millones de dólares, el retail es de suma importancia en el sector, manifestada en supermercados, tiendas por departamento, tiendas de descuento, equipamiento del hogar, productos farmacéuticos, entre otros. Debido a esto 500 de las mayores empresas en Perú, el sector comercio ocupó la tercera categoría de mayor importancia, después de actividades como petróleo-gas y servicios financieros [7].

El segmento empresarial peruano formal representa el $99,6 \%$ del total de empresas, siendo el 96,04\% microempresas, $3,44 \%$ las pequeñas y $0,12 \%$ las medianas; de las cuales el $87,9 \%$ se dedican a las actividades de comercio y servicios y el $12,1 \%$ a actividades productivas como la manufactura, construcción, agropecuario, minería y pesca. Donde las MYPES generan cerca de 59\% de la PEA ocupada, siendo considerada como fuente de empleo. En los últimos años (20152019) se ha incrementado el número de empresas formales a un ritmo de $8,4 \%$ promedio anual, sin embargo, aún existe el $36.9 \%$ de empresas informales no inscritas a la SUNAT [8].

En el contexto local, la empresa comercializadora "El Amiguito" SAC. dedicada al rubro de alimentos, lleva 14 años en el sector, se encuentra situada ubicada en Trujillo - Perú, actualmente es una zona donde existen 25 negocios dedicados al mismo rubro, por ende, siempre ha existido competencia entre estos.

Siendo este el principal problema identificado, se pudo analizar el comportamiento de los consumidores, llegando a la conclusión que estos tienen el poder absoluto de los precios, ya que si un negocio llega a vender un producto a un precio elevado, tiene la posibilidad de buscar un precio más cómodo en otro, cabe resaltar que una de las cualidades de estos, es que buscan un menor precio en vez de mejor calidad, debido a esto, la situación de los diversos negocios se ha visto afectados por los pocos ingresos que han logrado tener, ahora debido a la pandemia esta situación ha empeorado dando un duro golpe a todos los negocios, haciendo que algunos de estos cierren por falta de ingresos, de productos, de transporte o por el contagios de sus trabajadores. Por lo tanto, la presente investigación pretende obtener estrategias que permitan la captación de clientes para la diferenciación de la empresa en estudio ante sus competidores.

En referencia a lo antes mencionado se concluye que la formulación de estrategias de captación de clientes es de vital importancia para la empresa, debido a que posee un nivel alto de competencia y la identificación de estrategias le permitirá defenderse ante estos.

El objetivo de la investigación es Formular estrategias orientadas a la captación de clientes para la empresa El Amiguito SAC. en el año 2020, y como objetivos específicos:

-Analizar la situación actual de la empresa.

-Identificar estrategias de captación de clientes.

-Determinar las estrategias y los recursos necesarios.
-Estimar costos de estrategias propuestas y el impacto social y ambiental.

\section{Metodología}

\section{A. Diseño de la investigación}

\section{A.1 Tipo de investigación}

El tipo de investigación aplicada, longitudinal, prospectivo, dado que permite la visualización del desarrollo del futuro dependiendo de la acción del hombre [9].

\section{A.2 Población y muestra}

La población $(\mathrm{N})$, estuvo conformada por 100 clientes de la empresa. Debido a las restricciones sanitarias impuestas por el gobierno, se optó por un muestreo por conveniencia trabajando con un total de 20 clientes.

\section{A.3 Procedimiento}

Para el desarrollo de la investigación, se planteó 6 etapas, iniciando por una entrevista al Gerente General para la obtención de información acerca de la situación actual de la empresa. Se aplicó un cuestionario validado por juicio de expertos y verificada su confiabilidad mediante el alfa de Cronbach con un valor de 0.868 . Se recolecto información necesaria para el análisis de captación de clientes mediante un cuestionario elaborado. También se elaboró matrices que permitieron la identificación de estrategias filtradas mediante dimensiones implicadas.

Se planteó la hipótesis: Estrategias de captación de clientes contribuyen al posicionamiento de la empresa El Amiguito SAC. 2020, validándola mediante la formulación de ellas.

TABLA I

MODELO PLANTEADO

\begin{tabular}{|c|l|}
\hline Etapa & \multicolumn{1}{|c|}{ Modelo Planteado } \\
\hline 1 & Diagnóstico situacional de la empresa \\
\hline 2 & Análisis de factores internos y externos \\
\hline 3 & Identificación de estrategias de captación \\
\hline 4 & $\begin{array}{l}\text { Selección de estrategias, considerando las dimensiones: } \\
\text { satisfacción, fidelización y captación }\end{array}$ \\
\hline 5 & Especificación de estrategias por implementar \\
\hline 6 & Estimación de costos por estrategia planteada \\
\hline 7 & Determinación del impacto social y ambiental \\
\hline
\end{tabular}

En al Tabla I se denota las etapas para el desarrollo del modelo planteado.

\section{B. Estado del arte \\ B.1 Captación de clientes:}

El objetivo de la captación de clientes es introducirlos a la empresa, para esto existen diversas herramientas, como el análisis del marketing directo, que permiten captar a los mejores clientes con un menor costo. Algunas acciones de captación son: identificar el target, teniendo como objetivo encontrar el perfil del cliente. Y gestión de la primera venta, su objetivo es dar a conocer al cliente nuestra oferta de valor [10]. Así mismo, antes de captar clientes, se debe realizar un estudio 
del mercado, así poder obtener el sector más idóneo para realizar la comercialización [11]. Además, se detalla en cinco pasos cómo se logra la captación de clientes: Paso 1: Comprender el mercado, necesidades y deseos de los clientes, paso 2: Diseñar una estrategia de marketing orientada a los clientes seleccionados, paso 3: Realizar un programa de marketing donde se entregue valor al producto, paso 4: Construir una relación con los clientes, permitiendo la rentabilidad de la empresa y finalmente el paso 5: Lograr la captación de clientes [12].

\section{B.2 Satisfacción del cliente:}

La satisfacción del cliente es considerada un requisito fundamental para lograr posicionarnos en la mente de los clientes, llevándonos al mercado meta [13]. Se puede definir también como un estado de ánimo de una persona que compara el rendimiento recibido de un producto con sus expectativas [12].

B.3 Cliente:

Son las personas que tienen una necesidad de un producto o servicio que la empresa puede ofrecer, esta definición debería ser la base para cualquier empresa a nivel mundial [10].

\section{B.4 Fidelización del cliente:}

La fidelización del cliente como relaciones comerciales que mantiene el cliente con la entidad continuas o a largo plazo, la fidelización se produce cuando existe una buena actitud favorable entre empresa y cliente [14]. Es un concepto de marketing que posibilita la lealtad de un cliente hacia una marca, producto o servicio que ofrece una empresa, hacia un consumidor que compra de manera continua algún producto de la entidad. Se considera como estrategia la fidelización de los clientes, normalmente lo utilizan las empresas que tienen contacto directo con los clientes que consumen los productos que ofrecen, algunas entidades tienden a olvidarse de este paso, perdiendo clientela, impidiendo el éxito en el mercado [15].

\section{B.5 Marketing}

Marketing es considerado como cualquier acción por parte de las empresas, dirigidas especialmente para influir en el mercado anteriormente seleccionado [11]. También, identifica necesidades y deseos de los clientes; determinan los sectores que mejor pueden servir a la organización y se diseña los productos, servicios y programas adecuados para servir a esos mercados. Sin embargo, el objetivo del marketing es crear de forma rentable una satisfacción en los clientes al forjar relaciones de valor con los clientes importantes para la empresa [12].

\section{B.6 Estrategias}

La estrategia es definida como intermediario entre la empresa y su entorno, siendo su principal función determinar el uso de sus recursos para sus objetivos a largo plazo. Por ende, para lograr la formulación e implementación de la estrategia se debe tener en cuenta dos fases; en primer lugar, se debe tener el conocimiento del mercado y la organización alineadas a las circunstancias de la empresa. En segundo lugar, se toma en cuenta las características de liderazgo y capacidad organizativa para los implicados de la organización [15]. De modo que, una estrategia es positiva cuando la empresa está mejor con respecto a sus competidores que al inicio [16].

\section{RESULTADOS}

Etapal: Diagnóstico de la situación actual que presenta la empresa.

De las entrevistas realizadas se determinó la: Misión: Somos una empresa comercializadora de alimentos básicos, con el objetivo de satisfacer a nuestros clientes con la venta de productos de alta calidad provenientes de los mejores proveedores. Visión: Para el año 2030, aspiramos ser una empresa líder en la comercialización de productos de primera necesidad a nivel nacional con sucursales y almacenes en diversos lugares de La Libertad.

En esta primera etapa, también se aplicaron encuestas a clientes. TABLA II

ENCUESTAS APLICADAS

\begin{tabular}{|c|l|l|l|l|l|}
\hline Pregunta & Siempre & $\begin{array}{l}\text { Casi } \\
\text { siempre }\end{array}$ & A veces & $\begin{array}{l}\text { Casi } \\
\text { nunca }\end{array}$ & Nunca \\
\hline 1 & $20 \%$ & $55 \%$ & $25 \%$ & $0 \%$ & $0 \%$ \\
\hline 2 & $45 \%$ & $40 \%$ & $15 \%$ & $0 \%$ & $0 \%$ \\
\hline 3 & $65 \%$ & $35 \%$ & $0 \%$ & $0 \%$ & $0 \%$ \\
\hline 4 & $40 \%$ & $35 \%$ & $25 \%$ & $0 \%$ & $0 \%$ \\
\hline 5 & $15 \%$ & $60 \%$ & $25 \%$ & $0 \%$ & $0 \%$ \\
\hline 6 & $20 \%$ & $55 \%$ & $25 \%$ & $0 \%$ & $0 \%$ \\
\hline 7 & $5 \%$ & $10 \%$ & $45 \%$ & $30 \%$ & $0 \%$ \\
\hline 8 & $65 \%$ & $25 \%$ & $10 \%$ & $0 \%$ & $0 \%$ \\
\hline 9 & $15 \%$ & $55 \%$ & $30 \%$ & $0 \%$ & $0 \%$ \\
\hline 10 & $25 \%$ & $45 \%$ & 30 & $0 \%$ & $0 \%$ \\
\hline
\end{tabular}

En la Tabla II muestra las respuestas de todos los clientes encuestados, siendo las preguntas planteadas: Pregunta 1: ¿Se encuentra satisfecho con el servicio que brinda la empresa?, Pregunta 2: ¿Los productos recibidos cumplen con sus expectativas?, Pregunta 3: ¿La empresa cumple con el tiempo de entrega?, Pregunta 4: ¿Se encuentra satisfecho con el trato de los trabajadores?, Pregunta 5: ¿Los trabajadores tienen la capacidad de responder a sus necesidades?, Pregunta 6: ¿Considera correcto el precio pagado por los productos?, Pregunta 7: ¿Se siente cómodo con las instalaciones de la empresa?, Pregunta 8: ¿Le resulta fácil contactarse con el encargado de ventas?, Pregunta 9: ¿La empresa soluciona sus quejas o reclamos de manera óptima?, Pregunta 10: ¿La empresa le permite hacer sugerencias o reclamos, con la finalidad de mejorar el servicio?, analizando los diferentes resultados obtenidos, se pudo obtener una idea más clara de las necesidades y requisitos de los clientes, permitiéndole una ventaja ante sus competidores si se trabaja en los factores encontrados.

Etapa 2: Análisis interno y externo de la empresa 
En esta etapa, se desarrolló las siguientes matrices: FODA, EFE, EFI y PEYEA; para una mejor obtención de datos.

- Matriz FODA, permitió brindar información sobre las fortalezas y debilidades como las oportunidades y amenazas, obteniendo estrategias que permitan mejorar sus debilidades y afrontar sus amenazas.

TABLA III

MATRIZ FODA

\begin{tabular}{|c|c|}
\hline FORTALEZAS & DEBILIDADES \\
\hline $\begin{array}{l}\text { F1. Amplia experiencia en el } \\
\text { sector. }\end{array}$ & $\begin{array}{l}\text { D1. Falta de comunicación entre } \\
\text { áreas y encargados de procesos. }\end{array}$ \\
\hline F2. Productos si adulteración. & $\begin{array}{l}\text { D2. Inexistencia de planes de } \\
\text { contingencia ante riesgos o crisis. }\end{array}$ \\
\hline $\begin{array}{l}\text { F3. Facilidad de comunicación } \\
\text { de trabajadores y clientes. }\end{array}$ & $\begin{array}{l}\text { D3. Débil sistema de control de } \\
\text { inventarios. }\end{array}$ \\
\hline $\begin{array}{l}\text { F4. Cartera amplia de clientes } \\
\text { fieles. }\end{array}$ & $\begin{array}{l}\text { D4. Pérdida de datos e información } \\
\text { (documentados en folders, hojas). }\end{array}$ \\
\hline $\begin{array}{l}\text { F5. Productos con relación } \\
\text { calidad-precio. }\end{array}$ & $\begin{array}{l}\text { D5. Falta de programas de } \\
\text { capacitación a los trabajadores. }\end{array}$ \\
\hline $\begin{array}{l}\text { F6. Familiaridad de clientes con } \\
\text { la empresa. }\end{array}$ & $\begin{array}{l}\text { D6. Falta de uso de TI o recursos } \\
\text { digitales para ventas u otros procesos. }\end{array}$ \\
\hline F7. Personal con experiencia. & $\begin{array}{l}\text { D7. Punto de venta únicamente } \\
\text { presencial. }\end{array}$ \\
\hline $\begin{array}{l}\text { F8. Productos con enfoque en la } \\
\text { salud del usuario. }\end{array}$ & $\begin{array}{l}\text { D8. Personal limitado (se encargan de } \\
\text { varios cargos). }\end{array}$ \\
\hline $\begin{array}{l}\text { F9. Servicio de postventa, } \\
\text { servicio de transporte (taxi o } \\
\text { carretilla). }\end{array}$ & $\begin{array}{l}\text { D9. Poco capital para inversiones por } \\
\text { pérdida de ventas por el COVID. }\end{array}$ \\
\hline $\begin{array}{l}\text { F10. Proveedores con facilidad } \\
\text { de negociación. }\end{array}$ & D10. Infraestructura limitada. \\
\hline OPORTUNIDADES & AMENAZAS \\
\hline $\begin{array}{l}\text { O1. Leyes de reprogramación o } \\
\text { fraccionamiento de deudas. }\end{array}$ & $\begin{array}{l}\text { A1. Restricciones por aislamiento } \\
\text { social. }\end{array}$ \\
\hline $\begin{array}{l}\text { O2. Creación de programas de } \\
\text { facilidades de crédito (Reactiva } \\
\text { Perú). }\end{array}$ & $\begin{array}{l}\text { A2. Inestabilidad política (corrupción } \\
\text { y cambio de gobierno). }\end{array}$ \\
\hline $\begin{array}{l}\text { O3. Alianzas estratégicas con } \\
\text { otras empresas. }\end{array}$ & A3. Retroceso de la economía (crisis). \\
\hline $\begin{array}{l}\text { O4. Capacitación constante en } \\
\text { gestión, logística, etc. por el } \\
\text { Estado. }\end{array}$ & $\begin{array}{l}\text { A4. Disminución de la demanda (por } \\
\text { miedo a contagios o exposición } \\
\text { física). }\end{array}$ \\
\hline $\begin{array}{l}\text { O5. Incremento de demanda en } \\
\text { productos básicos (arroz, azúcar, } \\
\text { aceite). }\end{array}$ & $\begin{array}{l}\text { A5. Menor poder adquisitivo de los } \\
\text { clientes. }\end{array}$ \\
\hline $\begin{array}{l}\text { O6. TI para optimización y } \\
\text { mejora de procesos. }\end{array}$ & $\begin{array}{l}\text { A6. Aumento del costo en los } \\
\text { servicios. }\end{array}$ \\
\hline $\begin{array}{l}\text { O7. Mal servicio brindado por } \\
\text { parte de la competencia. }\end{array}$ & $\begin{array}{l}\text { A7. Competencia con mayor capital } \\
\text { de inversión y uso de TIC's. }\end{array}$ \\
\hline $\begin{array}{l}\text { O8. Alto poder de negociación } \\
\text { con proveedores. }\end{array}$ & $\begin{array}{l}\text { A8. Preferencia por productos en } \\
\text { supermercados. }\end{array}$ \\
\hline $\begin{array}{l}\text { O9. Conocimiento del lugar de } \\
\text { venta. }\end{array}$ & $\begin{array}{l}\text { A9. Pérdida de productos (robos, } \\
\text { clima, vencimiento). }\end{array}$ \\
\hline $\begin{array}{l}\text { O10. Competencia con productos } \\
\text { de adulterados. }\end{array}$ & $\begin{array}{l}\text { A10. Entrega de productos tardíos por } \\
\text { salidas interprovincial o } \\
\text { interdepartamental. }\end{array}$ \\
\hline
\end{tabular}

En la Tabla III, se muestra las fortalezas, (F1, F2, F3, F4, F5, F6, F7, F8, F9, F10), las debilidades, (D1, D2, D3, D4, D5, D6, D7, D8, D9, D10), las oportunidades, (O1, O2, O3, O4, O5, O6, O7, O8, O9, O10) y por último las amenazas, (A1, A2, A3, A4, A5, A6, A7, A8, A9, A10).
TABLA IV

MATRIZ FODA - ESTRATEGIAS

\begin{tabular}{|c|c|}
\hline ESTRATEGIAS FO (EXPLOTE) & $\begin{array}{c}\text { ESTRATEGIAS DO } \\
\text { (BUSQUE) }\end{array}$ \\
\hline \multirow{2}{*}{$\begin{array}{l}\text { Brindar capacitaciones e incentivos } \\
\text { al personal para su motivación (F3, } \\
\text { F7, F10, F9, 04). }\end{array}$} & $\begin{array}{c}\text { Contratación de personal } \\
\text { capacitado para el apoyo de los } \\
\text { demás procesos (D1, D8, 04, } \\
05) \text {. }\end{array}$ \\
\hline & $\begin{array}{c}\text { Implementación de medios } \\
\text { digitales para publicidad y } \\
\text { ventas (D6, D7, O4, 05, O6, } \\
\text { O7). }\end{array}$ \\
\hline \multirow[t]{2}{*}{$\begin{array}{l}\text { Desarrollo de un plan de marketing } \\
\text { para relacionar al cliente con la } \\
\text { marca, para identificación con la } \\
\text { empresa }(\mathrm{F} 1, \mathrm{~F} 3, \mathrm{~F} 4, \mathrm{~F} 6, \mathrm{O}, \mathrm{O} 9)\end{array}$} & $\begin{array}{c}\text { Buscar financiamiento externo } \\
\text { para cubrir demandas y obtener } \\
\text { ganancias por parte de } \\
\text { organizaciones el estado (D2, } \\
\text { D9, O1, 02). } \\
\end{array}$ \\
\hline & $\begin{array}{l}\text { Utilizar recursos TI gratuitos } \\
\text { por el momento para apoyo y } \\
\text { soporte de procesos (D1, D3, } \\
\text { D4, D6, O4, O5, O6, O7). }\end{array}$ \\
\hline $\begin{array}{l}\text { Brindar servicios adicionales a } \\
\text { clientes por frecuencia de compra, } \\
\text { recomendaciones, etc. (F2, F4, F6, } \\
\text { F8, F9, O5, O7, O9, O10). }\end{array}$ & $\begin{array}{l}\text { Realizar una campaña de } \\
\text { productos de baja demanda, } \\
\text { recuperando la inversión u } \\
\text { obteniendo ligeras ganancias } \\
\text { (D9, D10, O5 O9, O11). }\end{array}$ \\
\hline $\begin{array}{c}\begin{array}{c}\text { ESTRATEGIAS FA } \\
(\text { CONFRONTE })\end{array} \\
\end{array}$ & ESTRATEGIAS DA (EVITA) \\
\hline $\begin{array}{c}\text { Diseñar un modelo MPC para la } \\
\text { selección de proveedores para el } \\
\text { establecimiento de alianzas } \\
\text { estratégicas (F1, F2, F5, F8, F10, } \\
\text { A6, A10). }\end{array}$ & \multirow{2}{*}{$\begin{array}{c}\text { Implementar las consultas de } \\
\text { precios o servicios por medio de } \\
\text { llamadas o WhatsApp para } \\
\text { evitar el contacto físico directo } \\
\text { con los clientes y posibles } \\
\text { contagios (D6, D7, A1, A4, } \\
\text { A7). }\end{array}$} \\
\hline Realizar investigación y análisis del & \\
\hline $\begin{array}{c}\text { del producto }(\mathrm{F} 1, \mathrm{~F} 2, \mathrm{~F} 4, \mathrm{~A} 3, \mathrm{~A} 4 \\
\mathrm{A} 5, \mathrm{~A} 6, \mathrm{~A} 8)\end{array}$ & \multirow{2}{*}{$\begin{array}{l}\text { Negociaciones estratégicas para } \\
\text { la compra de productos, } \\
\text { determinando precio de } \\
\text { productos (D9, A5, A6). }\end{array}$} \\
\hline & \\
\hline (vendedor-cliente) (F8, A1, A4). & \multirow{2}{*}{$\begin{array}{c}\text { Implementar un sistema de } \\
\text { control de inventarios (gratuitos } \\
\text { momentáneamente por falta de } \\
\text { capital) para la optimización de } \\
\text { resultados área logística (D3, } \\
\text { D4, D6, A7). }\end{array}$} \\
\hline \multirow{2}{*}{$\begin{array}{l}\text { Implementación de palets en cada } \\
\text { pila de sacos y cambio de posiciones } \\
\text { de los productos, haciendo visibles } \\
\text { los de muestra, afirmando su } \\
\text { originalidad y resguardar los de } \\
\text { venta. (F2, F5, F8, A8, A9). }\end{array}$} & \\
\hline & \multirow{2}{*}{$\begin{array}{c}\text { Implementar un plan de } \\
\text { contingencia con medidas } \\
\text { específicas, en caso de afrontar } \\
\text { situaciones como pandemias, } \\
\text { desastres naturales, crisis } \\
\text { financiera o política, fraudes } \\
\text { con proveedores, entre otros } \\
\text { (D2, D9, A2, A3, A9, A10). }\end{array}$} \\
\hline $\begin{array}{l}\text { Realizar los pedidos a los } \\
\text { proveedores con tres meses de } \\
\text { anticipación (F1, F10, A1, A10). }\end{array}$ & \\
\hline
\end{tabular}

En la Tabla IV, se detalla las estrategias obtenidas para fortalezas-oportunidades, debilidades-oportunidades, fortaleza-amenaza y debilidad-amenaza.

- Matriz EFI, califica con valores desde 0.0 (sin importancia) hasta 1.0 (muy importante y con una clasificación de $1=$ debilidad mayor, $2=$ debilidad menor, $3=$ fortaleza menor y 4 =fortaleza menor. En la 
matriz de evaluación de los factores internos, ver Tabla V, da como resultado de 2.35 , lo cual, según la escala valorativa, se encuentra debajo del promedio fijado, esto quiere decir, que es una empresa con muchas debilidades internas.

TABLA V

MATRIZ DE FACTORES INTERNOS - EFI

\begin{tabular}{|c|c|c|c|}
\hline FACTORES CRÍTICOS & \multirow{2}{*}{ PESO } & \multirow{2}{*}{ CALIFICACIÓN } & \multirow{2}{*}{ VALOR } \\
\hline FORTALEZAS & & & \\
\hline $\begin{array}{l}\text { F1. Amplia experiencia en el } \\
\text { sector. }\end{array}$ & 0.04 & 2 & 0.08 \\
\hline F2. Productos si adulteración. & 0.03 & 1 & 0.03 \\
\hline $\begin{array}{l}\text { F3. Facilidad de comunicación de } \\
\text { trabajadores y clientes. }\end{array}$ & 0.05 & 3 & 0.15 \\
\hline $\begin{array}{l}\text { F4. Cartera amplia de clientes } \\
\text { fieles. }\end{array}$ & 0.05 & 2 & 0.1 \\
\hline $\begin{array}{l}\text { F5. Productos con relación calidad- } \\
\text { precio. }\end{array}$ & 0.04 & 1 & 0.04 \\
\hline $\begin{array}{l}\text { F6. Familiaridad de clientes con la } \\
\text { empresa. }\end{array}$ & 0.07 & 4 & 0.28 \\
\hline F7. Personal con experiencia. & 0.06 & 3 & 0.18 \\
\hline $\begin{array}{l}\text { F8. Productos con enfoque en la } \\
\text { salud del usuario. }\end{array}$ & 0.06 & 3 & 0.18 \\
\hline $\begin{array}{l}\text { F9. Servicio de postventa, servicio } \\
\text { de transporte (taxi o carretilla). }\end{array}$ & 0.07 & 2 & 0.14 \\
\hline $\begin{array}{l}\text { F10. Proveedores con facilidad de } \\
\text { negociación. }\end{array}$ & 0.02 & 1 & 0.02 \\
\hline Subtotal Fortalezas & 0.49 & & 1.20 \\
\hline \multicolumn{4}{|l|}{ DEBILIDADES } \\
\hline $\begin{array}{l}\text { D1. Falta de comunicación entre } \\
\text { áreas y encargados de procesos. }\end{array}$ & 0.09 & 4 & 0.36 \\
\hline $\begin{array}{l}\text { D2. Inexistencia de planes de } \\
\text { contingencia ante riesgos o crisis. }\end{array}$ & 0.04 & 1 & 0.04 \\
\hline $\begin{array}{l}\text { D3. Débil sistema de control de } \\
\text { inventarios. }\end{array}$ & 0.05 & 2 & 0.1 \\
\hline $\begin{array}{l}\text { D4. Pérdida de datos e información } \\
\text { (documentados en folders, hojas). }\end{array}$ & 0.06 & 3 & 0.18 \\
\hline $\begin{array}{l}\text { D5. Falta de programas de } \\
\text { capacitación a los trabajadores. }\end{array}$ & 0.04 & 1 & 0.04 \\
\hline $\begin{array}{l}\text { D6. Falta de uso de TI o recursos } \\
\text { digitales para ventas u otros } \\
\text { procesos. }\end{array}$ & 0.04 & 2 & 0.08 \\
\hline $\begin{array}{l}\text { D7. Punto de venta únicamente } \\
\text { presencial. }\end{array}$ & 0.02 & 1 & 0.02 \\
\hline $\begin{array}{l}\text { D8. Personal limitado (se encargan } \\
\text { de varios cargos). }\end{array}$ & 0.06 & 3 & 0.18 \\
\hline $\begin{array}{l}\text { D9. Poco capital para inversiones } \\
\text { por pérdida de ventas por el } \\
\text { COVID. }\end{array}$ & 0.04 & 2 & 0.08 \\
\hline D10. Infraestructura limitada. & 0.07 & 1 & 0.07 \\
\hline \multirow[t]{2}{*}{ Subtotal Debilidades } & 0.51 & & 1.15 \\
\hline & 1.00 & & 2.35 \\
\hline
\end{tabular}

- Matriz EFE, califica con valores desde 0.0 (sin importancia) hasta 1.0 (muy importante) y con una clasificación dependiendo la reacción que tiene la empresa con $1=$ deficiente, $2=$ nivel promedio, $3=$ arriba del promedio y $4=$ excelente. En la matriz de evaluación de los factores externos, ver Tabla VI, da como resultado de 2.22 , lo cual, según la escala valorativa, se encuentra debajo del promedio fijado, esto quiere decir, que es una empresa está en una posición deficiente con respecto a sus rivales, la empresa no aprovecha las oportunidades ni evitan las amenazas externas.

TABLA VI

MATRIZ DE FACTORES EXTERNOS - EFE

\begin{tabular}{|c|c|c|c|}
\hline & \multirow{2}{*}{ PESO } & \multirow{2}{*}{ CALIFICACIÓN } & \multirow{2}{*}{ VALOR } \\
\hline OPORTUNIDADES & & & \\
\hline $\begin{array}{l}\text { O1. Leyes de reprogramación o } \\
\text { fraccionamiento de deudas. }\end{array}$ & 0.03 & 1 & 0.03 \\
\hline $\begin{array}{l}\text { O2. Creación de programas de } \\
\text { facilidades de crédito (Reactiva Perú). }\end{array}$ & 0.03 & 1 & 0.03 \\
\hline $\begin{array}{l}\text { O3. Alianzas estratégicas con otras } \\
\text { empresas. }\end{array}$ & 0.05 & 2 & 0.10 \\
\hline $\begin{array}{l}\text { O4. Capacitación constante en gestión, } \\
\text { logística, etc. por el Estado. }\end{array}$ & 0.03 & 2 & 0.06 \\
\hline $\begin{array}{l}\text { O5. Incremento de demanda en } \\
\text { productos básicos (arroz, azúcar, } \\
\text { aceite). }\end{array}$ & 0.07 & 3 & 0.21 \\
\hline $\begin{array}{l}\text { O6. TI para optimización y mejora de } \\
\text { procesos. }\end{array}$ & 0.05 & 2 & 0.10 \\
\hline $\begin{array}{l}\text { O7. Mal servicio brindado por parte de } \\
\text { la competencia. }\end{array}$ & 0.06 & 3 & 0.18 \\
\hline $\begin{array}{l}\text { O8. Alto poder de negociación con } \\
\text { proveedores. }\end{array}$ & 0.04 & 3 & 0.12 \\
\hline O9. Conocimiento del lugar de venta. & 0.07 & 4 & 0.28 \\
\hline $\begin{array}{l}\text { O10. Competencia con productos de } \\
\text { adulterados. }\end{array}$ & 0.06 & 3 & 0.18 \\
\hline Subtotal Fortalezas & 0.49 & & 1.29 \\
\hline AMENAZAS & & & \\
\hline $\begin{array}{l}\text { A1. Restricciones por aislamiento } \\
\text { social. }\end{array}$ & 0.07 & 3 & 0.21 \\
\hline $\begin{array}{l}\text { A2. Inestabilidad política (corrupción y } \\
\text { cambio de gobierno). }\end{array}$ & 0.04 & 1 & 0.04 \\
\hline A3. Retroceso de la economía (crisis). & 0.06 & 1 & 0.06 \\
\hline $\begin{array}{l}\text { A4. Disminución de la demanda (por } \\
\text { miedo a contagios o exposición física). }\end{array}$ & 0.06 & 2 & 0.12 \\
\hline $\begin{array}{l}\text { A5. Menor poder adquisitivo de los } \\
\text { clientes. }\end{array}$ & 0.08 & 1 & 0.08 \\
\hline $\begin{array}{l}\text { A6. Aumento del costo en los } \\
\text { servicios. }\end{array}$ & 0.04 & 3 & 0.12 \\
\hline $\begin{array}{l}\text { A7. Competencia con mayor capital de } \\
\text { inversión y uso de TIC's. }\end{array}$ & 0.02 & 1 & 0.02 \\
\hline $\begin{array}{l}\text { A8. Preferencia por productos en } \\
\text { supermercados. }\end{array}$ & 0.06 & 2 & 0.12 \\
\hline $\begin{array}{l}\text { A9. Pérdida de productos (robos, } \\
\text { clima, vencimiento). }\end{array}$ & 0.04 & 2 & 0.08 \\
\hline $\begin{array}{l}\text { A10. Entrega de productos tardíos por } \\
\text { salidas interprovincial o } \\
\text { interdepartamental. }\end{array}$ & 0.04 & 2 & 0.08 \\
\hline \multirow[t]{2}{*}{ Subtotal Debilidades } & 0.51 & & 0.93 \\
\hline & 1.00 & & 2.22 \\
\hline
\end{tabular}

- Matriz PEYEA, califica a fuerza financiera (FF) y fuerza industrial (FI) desde 1 (la peor, lo que más afecta a la empresa) hasta 7 (la mejor, lo que menos afecta a la empresa) y a estabilidad del entorno (EE) y ventaja competitiva (VC) desde -1 (la mejor, lo que menos nos afecta) hasta -7 (la peor, lo que más nos afecta; obteniendo en $\mathrm{FF}=3.5, \mathrm{FI}=3.71, \mathrm{EE}=-4.50 \mathrm{Y}$ $\mathrm{VC}=-2.57$, ver Tabla VII. 
TABLA VII

MATRIZ DE POSICIÓN ESTRATÉGICA Y EVALUACIÓN DE LA ACCIÓN - PEYEA

\begin{tabular}{|c|c|c|c|}
\hline \multicolumn{2}{|c|}{$\begin{array}{c}\text { POSICIÓN EXTRATÉGICA } \\
\text { INTERNA }\end{array}$} & \multicolumn{2}{|c|}{$\begin{array}{c}\text { POSICIÓN ESTRATÉGICA } \\
\text { EXTERNA }\end{array}$} \\
\hline Fuerza Financiera (FF) & $\begin{array}{l}\text { Califiic } \\
\text { acion }\end{array}$ & $\begin{array}{l}\text { Estabilidad del Entorno } \\
\text { (EE) }\end{array}$ & $\begin{array}{l}\text { Calific } \\
\text { ación }\end{array}$ \\
\hline $\begin{array}{l}\text { Rendimiento sobre la } \\
\text { inversión }\end{array}$ & 3 & Cambios tecnológicos & -6 \\
\hline Apalancamiento & 2 & Tasa de inflación & -5 \\
\hline Liquidez & 4 & Variabilidad de la demanda & -5 \\
\hline Capital de trabajo & 3 & $\begin{array}{l}\text { Rango de precios de } \\
\text { productos de la competencia }\end{array}$ & -3 \\
\hline Flujo de efectivo & 4 & $\begin{array}{l}\text { Barreras de entrada al } \\
\text { mercado }\end{array}$ & -5 \\
\hline Rotación de efectivo & 6 & $\begin{array}{l}\text { Rivalidad/Presión } \\
\text { competitiva }\end{array}$ & -5 \\
\hline Rotación de inventarios & 3 & $\begin{array}{l}\text { Elasticidad de precios de la } \\
\text { demanda }\end{array}$ & -3 \\
\hline Utilidades por acción & 3 & $\begin{array}{l}\text { Presión de los productos } \\
\text { sustitutos }\end{array}$ & -4 \\
\hline TOTAL & 3.50 & TOTAL & -4.50 \\
\hline $\begin{array}{l}\text { Ventaja Competitiva } \\
\text { (VC) }\end{array}$ & $\begin{array}{l}\text { Calific } \\
\text { ación }\end{array}$ & Fuerza Industrial (FI) & $\begin{array}{l}\text { Calific } \\
\text { ación }\end{array}$ \\
\hline $\begin{array}{l}\text { Participación de } \\
\text { mercado }\end{array}$ & -1 & Potencial de crecimiento & 4 \\
\hline Calidad del producto & -1 & Potencial de utilidades & 5 \\
\hline $\begin{array}{l}\text { Ciclo de la vida del } \\
\text { producto }\end{array}$ & -1 & Estabilidad financiera & 4 \\
\hline Lealtad del consumidor & -5 & Grado de apalancamiento & 2 \\
\hline $\begin{array}{l}\text { Utilización de la } \\
\text { capacidad }\end{array}$ & -4 & Utilización de recursos & 2 \\
\hline $\begin{array}{l}\text { Conocimientos } \\
\text { tecnológicos prácticos }\end{array}$ & -4 & $\begin{array}{l}\text { Facilidad de entrada al } \\
\text { mercado }\end{array}$ & 4 \\
\hline $\begin{array}{l}\text { Control sobre } \\
\text { proveedores y } \\
\text { distribuidores }\end{array}$ & -2 & $\begin{array}{l}\text { Productividad/Utilización de } \\
\text { la capacidad }\end{array}$ & 5 \\
\hline TOTAL & -2.57 & TOTAL & 3.71 \\
\hline
\end{tabular}

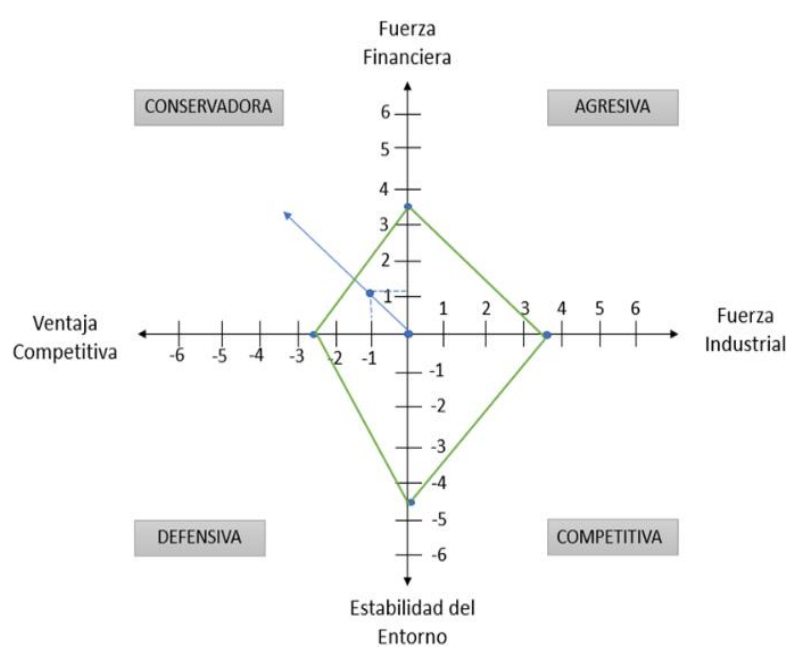

Fig. 1 Matriz de Posición estratégica y evaluación de la acción PEYEA
Del análisis presentado en la Fig. 1, se observa que se encuentra ubicado en el cuadrante conservadora $(\mathrm{X}=-1$; $\mathrm{Y}=$ 1.14) por ende se determina estrategias que cubran las siguientes características: penetración del mercado, desarrollar el mercado, desarrollar el producto y diversificación concéntrica.

\section{Etapa 3: Identificación de estrategias de captación}

Mediante el análisis a las diferentes matrices anteriormente detalladas, mostraron diferentes estrategias y características que deben poseer.

\section{FODA}

Estrategias Fortaleza - Oportunidades (FO):

- Brindar capacitaciones e incentivos al personal para su motivación (F3, F7, F10, F9, 04).

- Desarrollo de un plan de marketing para relacionar al cliente con la marca, para identificación con la empresa (F1, F3, F4, F6, O5, O9).

- Brindar servicios adicionales a clientes por frecuencia de compra, recomendaciones, etc. (F2, F4, F6, F8, F9, O5, O7, O9, O10).

Estrategias Debilidades - Oportunidades (DO):

- Contratación de personal capacitado para el apoyo de los demás procesos (D1, D8, 04, 05).

- Implementación de medios digitales para publicidad y ventas (D6, D7, O4, 05, O6, O7).

- Buscar financiamiento externo para cubrir demandas y obtener ganancias por parte de organizaciones el estado (D2, D9, O1, 02).

- Utilizar recursos TI gratuitos por el momento para apoyo y soporte de procesos (D1, D3, D4, D6, O4, O5, O6, O7).

- Realizar una campaña de productos de baja demanda, recuperando la inversión u obteniendo ligeras ganancias (D9, D10, O5 O9, O11).

Estrategias Fortalezas - Amenazas (FA):

- Diseñar un modelo MPC para la selección de proveedores para el establecimiento de alianzas estratégicas (F1, F2, F5, F8, F10, A6, A10).

- Realizar investigación y análisis del mercado para determinar precios del producto sin la pérdida de la calidad del producto (F1, F2, F4, A3, A4, A5, A6, A8).

- Implementar recursos para evitar el contagio en el proceso de compra (vendedor-cliente) (F8, A1, A4).

- Implementación de palets en cada pila de sacos y cambio de posiciones de los productos, haciendo visibles los de muestra, afirmando su originalidad y resguardar los de venta (F2, F5, F8, A8, A9).

- Realizar los pedidos a los proveedores con tres meses de anticipación (F1, F10, A1, A10).

Estrategias Debilidad - Amenaza (DA):

- Implementar las consultas de precios o servicios por medio de llamadas o WhatsApp para evitar el contacto físico directo con los clientes y posibles contagios (D6, D7, A1, A4, A7). 
- Negociaciones estratégicas para la compra de productos, determinando precio de productos (D9, A5, A6).

- Implementar un sistema de control de inventarios (gratuitos momentáneamente por falta de capital) para la optimización de resultados área logística (D3, D4, D6, A7).

- Implementar un plan de contingencia con medidas específicas, en caso de afrontar situaciones como pandemias, desastres naturales, crisis financiera o política, fraudes con proveedores, entre otros (D2, D9, A2, A3, A9, A10).

\section{MATRIZ MEFI}

En la matriz de evaluación de los factores internos (MEFI) da como resultado de 2.35 , lo cual, según la escala valorativa, se encuentra debajo del promedio fijado, esto quiere decir, que es una empresa con muchas debilidades internas.

\section{MATRIZ MEFE}

En la matriz de evaluación de los factores externos (MEFE) da como resultado de 2.22, lo cual, según la escala valorativa, se encuentra debajo del promedio fijado, esto quiere decir, que es una empresa está en una posición deficiente con respecto a sus rivales, la empresa no aprovecha las oportunidades ni evitan las amenazas externas.

\section{MATRIZ PEYEA}

Definición de estrategias

Cuadrante Conservadora:

- Penetración del mercado.

- Desarrollar el mercado.

- Desarrollar el producto.

- Diversificación concéntrica

Etapa 4: Selección de estrategias, considerando las dimensiones: satisfacción, fidelización y captación

En esta etapa se elaboró una matriz donde se filtró las estrategias que cumplen con las características y dimensiones mencionadas:

- Desarrollo de un plan de marketing para relacionar al cliente con la marca, para identificación con la empresa.

- Brindar adicionales a clientes por frecuencia de compra, recomendaciones, etc.

- Implementación de medios digitales para publicidad y ventas.

- Realizar una campaña de productos de baja demanda, recuperando la inversión u obteniendo ligeras ganancias.

- Implementar recursos para evitar el contagio en el proceso de compra (vendedor-cliente).

- Implementar las consultas de precios o servicios por medio de llamadas o WhatsApp para evitar el contacto físico directo con los clientes y posibles contagios.
TABLA VIII

MATRIZ DE SELECCIÓN DE ESTRATEGIAS

\begin{tabular}{|c|c|c|c|}
\hline $\begin{array}{l}\text { Dimensiones } \\
\text { Estrategias }\end{array}$ & Satisfacción & Captación & Fidelización \\
\hline $\begin{array}{l}\text { Desarrollo de un plan de } \\
\text { marketing para relacionar al } \\
\text { cliente con la marca, para } \\
\text { identificación con la } \\
\text { empresa. }\end{array}$ & $\mathbf{x}$ & $\mathbf{x}$ & $\mathbf{x}$ \\
\hline $\begin{array}{l}\text { Brindar } r \text { servicios } \\
\text { adicionales a clientes por } \\
\text { frecuencia de compra, } \\
\text { recomendaciones, etc. }\end{array}$ & $\mathbf{x}$ & $\mathbf{x}$ & $\mathbf{x}$ \\
\hline $\begin{array}{l}\text { Implementación de medios } \\
\text { digitales para publicidad y } \\
\text { ventas. }\end{array}$ & $\mathbf{x}$ & $\mathbf{x}$ & \\
\hline $\begin{array}{l}\text { Realizar una campaña de } \\
\text { productos de baja demanda, } \\
\text { recuperando la inversión u } \\
\text { obteniendo ligeras } \\
\text { ganancias. }\end{array}$ & & $\mathbf{x}$ & \\
\hline $\begin{array}{l}\text { Implementar recursos para } \\
\text { evitar el contagio en el } \\
\text { proceso de compra } \\
\text { (vendedor-cliente). }\end{array}$ & $\mathbf{x}$ & $\mathbf{x}$ & \\
\hline $\begin{array}{l}\text { Implementar las consultas } \\
\text { de precios o servicios por } \\
\text { medio de llamadas o } \\
\text { WhatsApp para evitar el } \\
\text { contacto físico directo con } \\
\text { los clientes y posibles } \\
\text { contagios. }\end{array}$ & $\mathbf{x}$ & $\mathbf{x}$ & \\
\hline
\end{tabular}

Etapa 5: Especificación de estrategias por implementar

En esta etapa se detalla los recursos que se van a requerir para la implementación de las estrategias.

Se realizará el desarrollo de un plan de marketing que incluye las diferentes características de las estrategias seleccionadas, para relacionar al cliente con la marca y para su identificación con la empresa. 
Definición de estrategias:

E1: Brindar servicios adicionales a clientes por frecuencia de compra, recomendaciones, etc.

E2: Implementación de medios digitales para publicidad y ventas.

E3: Realizar una campaña de productos de baja demanda, recuperando la inversión u obteniendo ligeras ganancias.

E4: Implementar recursos para evitar el contagio en el proceso de compra (vendedor-cliente).

E5: Implementar las consultas de precios o servicios por medio de llamadas o WhatsApp para evitar el contacto físico directo con los clientes y posibles contagios.

- Definir el desarrollo de la estrategia y recursos necesarios:

E1: Brindar servicios adicionales a clientes por frecuencia de compra compras, recomendaciones, etc.

Se les brindará la movilización de productos por medio de carretillas gratis solo cerca del local.

Se les brindará el servicio de carga.

Se les brindará el servicio de envase de 10 y $25 \mathrm{~kg}$ por saco.

Se les notificará de los nuevos productos adquiridos para venta y las características que posee.

Recursos: sacos de 10 y $25 \mathrm{~kg}$, carretilla, máquina industrial portátil de coser, hilo, balanza electrónica ventas.

E2: Implementación de medios digitales para publicidad y

Se realizará la creación de Facebook, Instagram e Instagram para la publicidad y venta de los productos.

Recursos: laptop o celular, internet.

E3: Realizar una campaña de productos de baja demanda, recuperando la inversión u obteniendo ligeras ganancias.

Brindar muestras gratuitas a los clientes de los productos de baja demanda, animando a su consumo.

Brindar descuentos y regalos (una lata de atún, una bolsa de fideos) por la compra del producto.

Recursos: bolsas para $1 \mathrm{~kg}$.

E4: Implementar recursos para evitar el contagio en el proceso de compra (vendedor-cliente).

Brindar mascarillas KN9, caretas al personal

Implementar una zona diferente en el local con alcohol líquido y en gel para los clientes y trabajadores.

Implementar un panel de vidrio para evitar el contagio directo en la zona de atención al cliente.

Recursos: mascarillas, caretas, alcohol de 70, gel antibacterial, panel de vidrio.

E5: Implementar las consultas de precios o servicios por medio de llamadas o WhatsApp para evitar el contacto físico directo con los clientes y posibles contagios.

Adquirir un celular para uso exclusivo de ventas de la empresa.

Recursos: celular, internet.
Realizar una calendarización de acciones para lograr el cumplimiento de las estrategias.

Determinar el presupuesto de la implementación.

\section{Etapa 6: Estimación de costos}

Para la estimación de costos que comprende la etapa 6 , nos da un total de S./ 6933.70 anual por la implementación de las estrategias, ver Tabla IX.

TABLA IX

COSTOS POR ESTRATEGIA

\begin{tabular}{|c|c|c|c|c|}
\hline Estrategia & Recurso & Cantidad & Precio & Total anual \\
\hline E1 & $\begin{array}{|lll|}\begin{array}{l}\text { Sacos } \\
\text { kg }\end{array} & & \\
\end{array}$ & 20 & $\mathrm{~S} / 1.00$ & $\mathrm{~S} / 240.00$ \\
\hline E1 & $\begin{array}{ll}\text { Sacos } & \mathrm{de} \\
25 \mathrm{~kg} & \end{array}$ & 20 & $\mathrm{~S} / 1.00$ & $\mathrm{~S} / 240.00$ \\
\hline E1 & Carretilla & 1 & $\mathrm{~S} / 55.00$ & $\mathrm{~S} / 55.00$ \\
\hline E1 & $\begin{array}{|ll|}\text { Máquina } & \\
\text { industrial } & \\
\text { portátil } & \text { de } \\
\text { coser } & \\
\end{array}$ & 1 & $\mathrm{~S} / 252.45$ & $\mathrm{~S} / 52.45$ \\
\hline E1 & Hilo & 2 & $\mathrm{~S} / 3.00$ & $\mathrm{~S} / 2.00$ \\
\hline E1 & $\begin{array}{l}\text { Balanza } \\
\text { electrónica }\end{array}$ & 1 & $\mathrm{~S} / 1,352.25$ & $\mathrm{~S} / 1,352.25$ \\
\hline E2 & Laptop & 1 & $\mathrm{~S} / 2,499.00$ & $\mathrm{~S} / 2,499.00$ \\
\hline E2 - E5 & Internet & 1 & $\mathrm{~S} / 50.00$ & $\mathrm{~S} / 600.00$ \\
\hline $\mathbf{E 3}$ & \begin{tabular}{|l|} 
Bolsas para 1 \\
$\mathrm{~kg}$
\end{tabular} & 1 & $\mathrm{~S} / 2.00$ & $\mathrm{~S} / 24.00$ \\
\hline E4 & Mascarillas & 1 & $\mathrm{~S} / 20.00$ & $\mathrm{~S} / 240.00$ \\
\hline E4 & Caretas & 4 & $\mathrm{~S} / 2.00$ & $\mathrm{~S} / 8.00$ \\
\hline E4 & Alcohol de 70 & 4 & $\mathrm{~S} / 9.00$ & $\mathrm{~S} / 432.00$ \\
\hline E4 & $\begin{array}{l}\text { Gel } \\
\text { antibacterial }\end{array}$ & 3 & $\mathrm{~S} / 9.00$ & $\mathrm{~S} / 324.00$ \\
\hline E4 & $\begin{array}{|ll|}\begin{array}{l}\text { Panel } \\
\text { vidrio }\end{array} & \text { de } \\
\end{array}$ & 1 & $\mathrm{~S} / 60.00$ & $\mathrm{~S} / 60.00$ \\
\hline E3 - E5 & Celular & 1 & S/535.00 & $\mathrm{S} / 535.00$ \\
\hline \multicolumn{4}{|c|}{ Total } & S/6,933.70 \\
\hline
\end{tabular}


Etapa 7: Determinación del impacto social y ambiental

TABLA X

MATRIZ DE IMPACTO SOCIAL Y AMBIENTAL

\begin{tabular}{|c|c|c|c|c|}
\hline \multirow{2}{*}{$\begin{array}{l}\text { DIMENSÓN } \\
\text { ESTRATEGIA }\end{array}$} & \multicolumn{2}{|l|}{ SOCIAL } & \multicolumn{2}{|c|}{ AMBIENTAL } \\
\hline & Positiva & Negativa & Positiva & Negativa \\
\hline $\begin{array}{l}\text { E1: Brindar } \\
\text { servicios } \\
\text { adicionales a } \\
\text { clientes por } \\
\text { frecuencia de } \\
\text { compra, } \\
\text { recomendacion } \\
\text { es, etc. }\end{array}$ & $\begin{array}{l}\text { Mejora el } \\
\text { poder } \\
\text { adquisitivo } \\
\text { de los } \\
\text { clientes. }\end{array}$ & & $\begin{array}{l}\text { Utilización } \\
\text { de } \\
\text { carretillas } \\
\text { reemplaza } \\
\text { ndo carros, } \\
\text { disminuye } \\
\text { ndo el } \\
\text { dióxido de } \\
\text { carbono } \\
\text { emanado } \\
\text { de los } \\
\text { autos. } \\
\end{array}$ & \\
\hline $\begin{array}{l}\text { E2: } \\
\text { Implementación } \\
\text { de medios } \\
\text { digitales para } \\
\text { publicidad y } \\
\text { ventas. }\end{array}$ & $\begin{array}{l}\text { Evita robos } \\
\text { al momento } \\
\text { de ir a la } \\
\text { tienda. } \\
\text { Evita } \\
\text { disturbios } \\
\text { y/o } \\
\text { conflictos. } \\
\end{array}$ & & $\begin{array}{l}\text { Evita } \\
\text { aglomeraci } \\
\text { ón y } \\
\text { posibles } \\
\text { contagios. }\end{array}$ & \\
\hline $\begin{array}{l}\text { E3: Realizar } \\
\text { una campaña de } \\
\text { productos de } \\
\text { baja demanda, } \\
\text { recuperando la } \\
\text { inversión u } \\
\text { obteniendo } \\
\text { ligeras } \\
\text { ganancias. }\end{array}$ & $\begin{array}{l}\text { Mejora la } \\
\text { posibilidad } \\
\text { de compra } \\
\text { de los } \\
\text { clientes. }\end{array}$ & & $\begin{array}{l}\text { Evita } \\
\text { pérdidas y } \\
\text { posibles } \\
\text { residuos } \\
\text { generando } \\
\text { contamina } \\
\text { ción. } \\
\text { (productos } \\
\text { desechado } \\
\text { s) } \\
\end{array}$ & \\
\hline $\begin{array}{l}\text { E4: } \\
\text { Implementar } \\
\text { recursos para } \\
\text { evitar el } \\
\text { contagio en el } \\
\text { proceso de } \\
\text { compra } \\
\text { (vendedor- } \\
\text { cliente). }\end{array}$ & $\begin{array}{l}\text { Evita } \\
\text { disgusto de } \\
\text { vecinos por } \\
\text { falta de } \\
\text { implementa } \\
\text { ción de } \\
\text { recursos. } \\
\text { Evitar } \\
\text { cierre del } \\
\text { local. }\end{array}$ & & $\begin{array}{l}\text { Evitar } \\
\text { contagios } \\
\text { a clientes } \\
\text { y } \\
\text { propagació } \\
\text { n de virus. }\end{array}$ & $\begin{array}{l}\text { Residuos } \\
\text { de envases } \\
\text { de alcohol } \\
\text { y alcohol } \\
\text { en gel. }\end{array}$ \\
\hline $\begin{array}{l}\text { E5: } \\
\text { Implementar las } \\
\text { consultas de } \\
\text { precios o } \\
\text { servicios por } \\
\text { medio de } \\
\text { llamadas o } \\
\text { WhatsApp para } \\
\text { evitar el } \\
\text { contacto físico } \\
\text { directo con los } \\
\text { clientes y } \\
\text { posibles } \\
\text { contagios }\end{array}$ & $\begin{array}{l}\text { Mejora la } \\
\text { relación } \\
\text { con los } \\
\text { vecinos, } \\
\text { para evitar } \\
\text { aglomeraci } \\
\text { ones. }\end{array}$ & & $\begin{array}{l}\text { Evitar } \\
\text { contagios } \\
\text { masivos y } \\
\text { propagació } \\
\text { n de virus. }\end{array}$ & \\
\hline
\end{tabular}

En la Tabla $X$ se puede analizar que en la mayoría de estrategias implementadas resulta positiva en la sociedad y el ambiente.

Para la estrategia 4, los trabajadores realizan buenas prácticas con respecto al uso de mascarillas, no utilizan las quirúrgicas, ellos utilizan mascarillas de tela, las cuales son lavadas para su nuevo uso, por lo tanto, evitan la contaminación; sin embargo, también genera desechos debido a los envases de alcohol.

\section{DISCUSIÓN}

La presente investigación presentó ciertas limitaciones en la recolección de datos, no se permitió la presencia física del investigador con cliente, para evitar algún tipo de contagio, ya que, se encuentran expuestos por el virus en la zona, sin embargo, se apoyó en los recursos tecnológicos. El 20\% de los clientes encuestados exponen que se encuentran satisfechos con el servicio brindado por la empresa, un 55\% casi siempre; sin embargo, los resultados obtenidos pueden tener cierto margen de error, ya que se realizó un muestreo por conveniencia, siendo un total de 20 clientes encuestados.

Se pudo analizar como la pandemia afecta a la empresa ya que no contaban con plan de contingencia además de existir poca inversión por la crisis en la que atraviesan, ver Tabla III, esto confirma la investigación de [1].

La investigación reconoce la importancia de la transformación digital, ver Tabla VIII, frente a la crisis sanitaria actualmente atravesada, teniendo en cuenta la posibilidad de inversión por parte de la empresa, estos resultados analizados, confirman la investigación de [2].

Si bien es cierto, la empresa no se encuentra automatizada, sus trabajadores no tienen conocimiento del manejo de sistemas complejos, ver Tabla III, por lo cual es complicado digitalizar a la empresa para el apoyo de sus actividades, esto contrasta a la investigación de [3], debido a que se pueden plantear otras estrategias que no le generen gastos elevados a la empresa a cambio de poco beneficio.

Se detalla la importancia de la inversión en recursos antiCOVID y medios digitales para promoción productos ofrecidos por la empresa, permitiendo la captación y fidelización de clientes, ver Tabla IV y VIII, esto concuerda con la investigación planteada por [4].

Se emplearon estrategias que permitan cumplir con las necesidades del público y lograr su satisfacción, ver Tabla II, además se busca que sean accesibles para la empresa, ver tabla 8 , esto confirma la investigación presentada por [5].

La implicancia metodológica de la investigación radica en que puede servir como antecedente para futuras investigaciones, ya que es una metodología propia, ver Tabla I, donde se detalla el proceso que se siguió para lograr identificar las estrategias orientadas a la captación de clientes, iniciando por un análisis con diferentes matrices que brindaron diversas estrategias, sin embargo, no todas cumplían con el logro del objetivo, por ello, se filtraron para lograr obtener las estrategias que cumplían con la finalidad de la investigación. 


\section{CONCLUSIONES}

Se formuló estrategias orientadas para la captación de clientes de la empresa en estudio, ver Tabla VIII:

- Brindar servicios adicionales a clientes por frecuencia de compra y recomendaciones, entre otras características.

- Implementación de medios digitales para publicidad y ventas.

- Realizar una campaña de productos de baja demanda, recuperando la inversión u obteniendo ligeras ganancias.

- Implementar recursos para evitar el contagio en el proceso de la venta (vendedor - cliente).

- Implementar las consultas de precios o servicios por medio de llamadas o el uso del WhatsApp para evitar el contacto físico directo con los clientes y posibles contagios.

Se realizó un diagnóstico situacional actual de la empresa, mediante la elaboración de la matriz FODA, ver Tabla III, donde se pudo analizar y conocer cómo la empresa trabaja, tanto interno como externo, además se elaboró la matriz de evaluación de los factores internos, ver Tabla $\mathrm{V}$, dando como resultado de 2.35, lo cual, se encuentra debajo del promedio fijado, esto quiere decir, que es una empresa con muchas debilidades internas y la matriz de evaluación de los factores externos, ver Tabla VI, dando como resultado de 2.22, lo cual, según la escala valorativa, se encuentra debajo del promedio fijado, esto quiere decir, que es una empresa está en una posición deficiente con respecto a sus rivales, la empresa no aprovecha las oportunidades ni evitan las amenazas externas y finalmente se planteó la matriz PEYEA, ver Tabla VII, donde mediante el análisis y desarrollo, se obtuvo estrategias del cuadrante conservadora; mediante todo estos datos obtenidos, se pudo concluir que tienen poca información y/o son ajenos al tema con respecto a la formulación de estrategias adecuadas para lograr la captación de los clientes ya que existen alrededor de 25 negocios y proponer estrategias de captación sería de gran ventaja para lograr un mejor posicionamiento ante sus competidores.

Se identificó las estrategias de captación del cliente, como se observa en la Tabla VIII, considerando los aspectos que abarcan a la captación, satisfacción y fidelización del cliente.

Se determinó las estrategias de captación de clientes y los recursos necesarios para su implementación, como se demuestra en la Tabla IX.

Se estimo los costos ascendentes a S./ 6933.70 anuales, para la implementación de las estrategias propuestas, ver Tabla IX. Y finalmente se detalló el impacto de las estrategias implementadas en el medio ambiente y en la sociedad, ver Tabla X.

\section{AGRADECIMIENTO}

Expreso mi agradecimiento al Ing. Odar R. Florián Castillo por brindarme la ayuda, oportunidad y confianza para presentar este artículo.

A mi familia, en especial a mi madre por su apoyo incondicional.

Y al Sr. Víctor Chávez Gerente General y al señor Ulises Dominguez encargado de ventas de la empresa El Amiguito SAC, que brindó la información necesaria para el desarrollo del artículo.

\section{REFERENCIAS}

[1] Brauner, D., Reichert, F., Muniz, R., Zen, A., Menezes, D. Closs L., Carraro, W., Ruppenthal, C., Müller F. (2020). AN ENGAGED UNIVERSITY: RESCUING SMEs DURING THE COVID-19 CRISIS. Revista de Administração de Empresas

[2] Fierro, E.. (2021). La gestión del cambio, la colaboración virtual y la agilidad estratégica organizacional de empresas mexicanas ante los impactos por el COVID-19. Nova scientia.

[3] Martinez, A. (2021). Impacto de la COVID-19 en la producción, empleo y digitalización de empresas en Guanajuato: una primera aproximación. Nova scientia.

[4] Silva, D., Miranda, A., Hoffmann, V., (2021). IViva ou Deixe Morrer: estratégias para o enfrentamento da COVID-19 sob a perspectiva empresarial em São Luís do Maranhão, Brasil.. Revista Brasileira de Pesquisa em Turismo

[5] Fandos, J. C., Sánchez, J., Moliner, M. Á., Estrada, M. (2011). La lealtad del consumidor en el sector financiero. Innovar.

[6] https://www.cepal.org/es/comunicados/comercio-internacionalamerica-latina-caribe-caera-23-2020-debido-efectos-la-pandemia

[7] La Cámara de Comercio de Lima. (2019). Perspectivas del sector comercio y las oportunidades para hacer negocios [Archivo de video]. Recuperado de: https://gestion.pe/gestion-tv/perspectivas-sectorcomercio-oportunidades-negocios-265912-noticia/?ref=gesr

[8] Ministerio de produccion (2019). Estadisticas MIPYME. Recuperado de: https://ogeiee.produce.gob.pe/index.php/en/shortcode/estadisticaoee/estadisticas-mipyme

[9] Gil, J. (2005). Prospectiva: ¡Una Herramienta Poderosa!. Revista Científica General José María Córdova.

[10] Barquero, J. (2007). Marketing de clientes ¿Quién se ha llevado a mi cliente?. Recuperado de: https://ebookcentral.proquest.com/lib/upnpe/reader.action?docID=319 $4798 \&$ query $=$ barquero $+2007 \#$

[11] Martínez, J. y Jiménez, E., (2001), Marketing. Recuperado de: https://ebookcentral.proquest.com/lib/upnpe/reader.action?docID=318 6896

[12] Armstrong, G. y Kotler, P., (2013). Fundamentos del Marketing. Recuperado https://frrq.cvg.utn.edu.ar/pluginfile.php/14584/mod_resource/content/ 1/Fundamentos\%20del\%20Marketing-Kotler.pdf

[13] Alpízar, A. y Hernández, L. (2015), Estudio de mercado para determinar el nivel de satisfacción del cliente en Estelaris Mueblerías, 2015. (tesis de pregrado). Universidad autónoma de la ciudad de México

[14] Mesén, V. (2011). Fidelización de clientes: concepto y perspectiva contable. Tec Empresarial.

[15] Vivanco, R. (2019). Marketing relacional enfocado a la fidelización de los clientes del restaurante Mar Criollo, Lima, 2019. (tesis de pregrado). Universidad Norbert Wiener, Lima, Perú.

[16] González, B., \& Atencio, E. (2010). Estrategias de servicio: elementos para el éxito empresarial. Multiciencias. 